\title{
Low- $Z$ gas stripper as an alternative to carbon foils for the acceleration of high-power uranium beams
}

\author{
H. Okuno, ${ }^{*}$ N. Fukunishi, A. Goto, H. Hasebe, H. Imao, O. Kamigaito, M. Kase, H. Kuboki, Y. Yano, and S. Yokouchi \\ RIKEN, Nishina Center for Accelerator-Based Science, 2-1, Hirosawa, Wako, Saitama 351-0198, Japan
}

A. Hershcovitch

Brookhaven National Laboratory, Upton, New York 11973, USA

(Received 25 December 2010; published 23 March 2011)

\begin{abstract}
The RIKEN accelerator complex started feeding the next-generation exotic beam facility radioisotope beam factory (RIBF) with heavy-ion beams from 2007 after the successful commissioning of RIBF at the end of 2006. Many improvements made from 2007 to 2010 were instrumental in increasing the intensity of various heavy-ion beams. However, the available beam intensity of very heavy ion beams, especially uranium beams, is far below our goal of $1 \mathrm{p} \mu \mathrm{A}\left(6 \times 10^{12}\right.$ particles/s). In order to achieve this goal, upgrade programs are already in progress; the programs include the construction of a new $28-\mathrm{GHz}$ superconducting electron cyclotron resonance ion source and a new injector linac. However, the most serious problem, that of a charge stripper for high-power uranium beams, still remains unsolved, despite extensive research and development work using large foils mounted on a rotating cylinder and a $\mathrm{N}_{2}$ gas stripper. A gas stripper is free from problems related to lifetime, though the equilibrium charge state in this stripper is considerably lower than that in a carbon foil, owing to the absence of the density effect. Nevertheless, the merits of gas strippers motivated us to develop a low- $Z$ gas stripper to achieve a higher equilibrium charge state even in gases. We measured the electron-loss and electron-capture cross sections of uranium ions in $\mathrm{He}$ gas as a function of their charge state at 11, 14, and $15 \mathrm{MeV} /$ nucleon. The equilibrium charge states extracted from the intersection of the lines of the two cross sections were promisingly higher than those in $\mathrm{N}_{2}$ gas by more than 10 . Simple simulations of charge development along the stripper thickness were performed by assuming the measured cross sections. The simulation results show that about $1 \mathrm{mg} / \mathrm{cm}^{2}$ of $\mathrm{He}$ gas should be accumulated to achieve a charge state higher than that of $\mathrm{N}_{2}$ gas, notwithstanding the difficulty in accumulation of this helium amount owing to its fast dispersion. However, we now believe that the following two solutions can overcome this difficulty: a gas cell with a very large differential pumping system and a gas cell with a plasma window. Their merits and demerits are discussed in the paper.
\end{abstract}

DOI: 10.1103/PhysRevSTAB.14.033503

PACS numbers: 29.20.dg, 29.25.Pj, 34.50.Fa

\section{INTRODUCTION}

The RIKEN Nishina Center for Accelerator-Based Science constructed the radioisotope beam factory (RIBF) [1] with the aim of realizing a next-generation facility that can provide the world's most intense RI beams at energies of several hundred $\mathrm{MeV} /$ nucleon over the entire range of atomic masses. The RIBF requires an accelerator complex that can accelerate ions over the entire range of masses and deliver $80-\mathrm{kW}$ uranium beams at an energy of $345 \mathrm{MeV} /$ nucleon. Figure 1 shows a bird's-eye view of the RIBF. The structure on the left is the old facility that was completed in 1990. Many fruitful experiments were carried out with light-ion RI beams using the

\footnotetext{
*okuno@riken.jp

Published by American Physical Society under the terms of the Creative Commons Attribution 3.0 License. Further distribution of this work must maintain attribution to the author(s) and the published article's title, journal citation, and DOI.
}

four-sector K540-MeV RIKEN ring cyclotron (RRC) [2] with two injectors, RIKEN linear accelerator (RILAC) [3] and the azimuthally varying field (AVF) cyclotron $(\mathrm{K} 70 \mathrm{MeV})$ [4], because the RRC can accelerate relatively light ions up to $100 \mathrm{MeV} /$ nucleon, which is the lower limit for RI beam production. In order to extend the mass range for RI beam production up to uranium, three ring cyclotrons, the fixed-frequency ring cyclotron (fRC) [5], intermediate-stage ring cyclotron (IRC) [6], and superconducting ring cyclotron (SRC) [7] were designed and constructed as energy boosters for the RRC. The SRC is the first ring cyclotron in the world that uses superconducting sector magnets with the largest bending power. The accelerated beams are transported to an in-flight RI beam separator BigRIPS [8].

The design and construction of the RIBF accelerators started in 1997, and a building for housing the accelerators completed construction at the end of March 2003. In November 2005, we reached an important milestone: The superconducting sector magnets for the SRC were 




FIG. 1. Bird's-eye view of RIBF. Two injectors, RIKEN linear accelerator (RILAC) and the AVF cyclotron (K70 MeV), are followed by four booster cyclotrons: RIKEN ring cyclotron [(RRC), K540 MeV], fixed-frequency ring cyclotron (fRC), intermediate-stage ring cyclotron (IRC), and superconducting ring cyclotron (SRC). The accelerated beams are transported to an in-flight RI beam separator BigRIPS.

successfully excited at the maximum field level. The first beam was obtained on December 28, $2006[9,10]$. Many improvements were made to the accelerators for increasing beam intensity and commissioning new beam species to meet the requirements of different experiments. Table I lists the beams accelerated thus far. These beams were used in many nuclear physics experiments such as the discovery of 45 new isotopes [11] and studies of the halo structure and large deformation of extremely neutron rich Ne isotopes $[12,13]$. Our goal is to achieve a beam intensity of $1 \mathrm{p} \mu \mathrm{A}\left(6 \times 10^{12}\right.$ particles $\left./ \mathrm{s}\right)$ for the entire atomic range. We achieved the target intensity for $\mathrm{He}$ and $\mathrm{O}$ ions and about one-fourth of the target intensity for $\mathrm{Ca}$ ion. However, the intensity of uranium beams is still very low and drastic measures are required to achieve the target intensity.

This paper reports that a low- $Z$ gas stripper presents a new possibility to resolve a serious problem related to a charge stripper, which plays an essential role in high-power uranium beam acceleration. In Sec. II, we provide an overview of the upgrade program for increasing the intensity of the uranium beam, which involves construction of a new ion source and a new injector. We also report the status of the research and development $(\mathrm{R} \& \mathrm{D})$ work on the charge stripper, which has thus far not been very fruitful. In Sec. III, we discuss equilibrium charge state in low- $Z$

TABLE I. Beams accelerated at the RIBF accelerator complex with the maximum beam intensity achieved. The beam intensities are expressed in pnA $\left(6 \times 10^{9}\right.$ particles $\left./ \mathrm{sec}\right)$.

\begin{tabular}{lcrl}
\hline \hline Ion & $\begin{array}{c}\text { Energy } \\
(\mathrm{MeV} / \text { nucleon })\end{array}$ & $\begin{array}{c}\text { Intensity } \\
(\mathrm{pnA})\end{array}$ & \multicolumn{1}{c}{ Date } \\
\hline pol-d & 250 & 120 & May 2009 \\
$\mathrm{H}^{4} \mathrm{e}$ & 320 & 1000 & Oct 2009 \\
${ }^{14} \mathrm{~N}$ & 250 & 80 & May 2009 \\
${ }^{8} \mathrm{O}$ & 345 & 1000 & Jun 2010 \\
${ }^{48} \mathrm{Ca}$ & 345 & 230 & May 2010 \\
${ }^{86} \mathrm{Kr}$ & 345 & 30 & Nov 2007 \\
${ }^{238} \mathrm{U}$ & 345 & 0.8 & Dec 2009 \\
\hline \hline
\end{tabular}

gas strippers and report the experimental results for the equilibrium charge state extracted from the intersection of the electron-loss and electron-capture cross sections measured in $\mathrm{He}$ gas at 11,14 , and $15 \mathrm{MeV} /$ nucleon as a function of the ion charge. In Sec. IV, we present the simulation results for charge development along the thickness of the He gas. Further, we propose two solutions for overcoming the difficulty in accumulation of $1-\mathrm{mg} / \mathrm{cm}^{2}$ He gas.

\section{INCREASING THE URANIUM BEAM INTENSITY AND THE RELATED CHARGE STRIPPER PROBLEM}

From our operational experience mentioned in the previous section, the key issues to be addressed for increasing the intensity of uranium beams are as follows. First, generation of beams with higher intensity from the ion source is required. Nakagawa et al. are currently developing a new $28-\mathrm{GHz}$ superconducting electron cyclotron resonance (ECR) ion source, which is designed to have a plasma volume as large as $1100 \mathrm{~cm}^{3}[14,15]$. An important feature of this source is that its coil system is designed to provide a flat magnetic field distribution in the central region, by exciting the solenoids independently. This ion source is expected to produce $\mathrm{U}^{35+}$ ions at an intensity greater than $15 \mathrm{p} \mu \mathrm{A}$, which is necessary to obtain $1-\mathrm{p} \mu \mathrm{A}$ beams from the SRC. The coil was successfully excited to the designed level in October 2008. The testing of the ECR source began in April 2009; the 18-GHz mode was used for this test because a $28-\mathrm{GHz}$ power supply was not available then. The intensity of uranium beams reached $10 \mathrm{e} \mu \mathrm{A}$, which is about 5 times that of the beams from the previously used ion sources. The ion source will be moved to upstream of a new injector and will be tested in the $28-\mathrm{GHz}$ mode in the autumn of 2010. Another important issue in increasing the uranium beam intensity is the necessity of a new injector to prevent emittance growth due to space charge forces during the acceleration of ion beams from the new powerful ion source. A new injector has been designed to efficiently accelerate ions with a mass-to-charge ratio of 7 and is 
aimed at heavy ions such as ${ }^{84} \mathrm{Kr}^{13+},{ }^{136} \mathrm{Xe}^{20+}$, and ${ }^{238} \mathrm{U}^{35+}$, up to an energy of $680 \mathrm{keV} /$ nucleon [16]. This injector mainly consists of a radio-frequency quadrupole (RFQ) linac based on the four-rod structure and three drift-tube linacs (DTLs) based on a quarter-wavelength resonator. All the main components have already been installed, and the excitation test of all the accelerator tanks has been performed with the aim of starting the beam commissioning from mid-December 2010.

The final key issue is the development of a charge stripper with a long lifetime, which is still an open problem. Figure 2 shows the acceleration scheme for uranium beams using two strippers. The uranium beams are accelerated using RFQ, RILAC, RRC, fRC, IRC, and SRC. The first stripper is located behind the RRC, with an energy of $11 \mathrm{MeV} /$ nucleon, and the second one is located behind the fRC, with an energy of $51 \mathrm{MeV} /$ nucleon. Carbon foils are used in both strippers. The typical thicknesses of the foils for the first and second strippers are $300 \mu \mathrm{g} / \mathrm{cm}^{2}$ and $17 \mathrm{mg} / \mathrm{cm}^{2}$, respectively. The use of the first stripper involves a serious problem. Carbon foils commercially available from ACF-Metals [17] are used for the first stripper. Their typical lifetime is approximately $12 \mathrm{~h}$ at an intensity of 1 e $\mu \mathrm{A}$. Carbon foils of the same thickness are being developed at RIKEN, the quality of which is getting closer to that of the commercially available ones [18]. The currently available beam intensities do not pose any problems. However, in the future, with the completion of the aforementioned upgrade programs, the intensity of uranium beams will increase more than 100 -fold, thereby leading to the requirement much stronger strippers.

Accordingly, intense $R \& D$ programs focusing on upgrading the first stripper were initiated in 2008. We first started conducting irradiation tests on the large foil mounted on the rotating cylinder developed by Ryuto et al. [19] to expand the irradiation area, expecting to realize a lifetime about 60 times longer than that of the fixed carbon foils. We placed a $100-\mathrm{mm}$ diameter foil on the cylinder, which can rotate in beam vacuum. The first



FIG. 2. Acceleration scheme for uranium beams at RIBF. The uranium beams from an $18-\mathrm{GHz}$ electron cyclotron resonance ion source (ECRIS) are accelerated using two linear accelerators, radio-frequency quadrupole linac (RFQ) and RILAC, and four ring cyclotrons, RRC, fRC, IRC, and SRC, for RI beam production in BigRIPS. Two charge strippers are located downstream of the first and second cyclotrons, RRC and fRC, respectively. The numbers in the brackets below the cyclotrons denote the extraction energies in $\mathrm{MeV} /$ nucleon. sample tested in 2008 broke in as short a time as about $15 \mathrm{~min}$. We performed some tests to determine why the rotating foil broke so soon. We found that a very slowly $(0.05 \mathrm{rpm})$ rotating foil can survive for more than $38 \mathrm{~h}$ at an intensity of $1.7 \mathrm{e} \mu \mathrm{A}$ without experiencing significant damage. However, we also found that the beam intensity behind the stripper changes periodically, and hence, we could not tune the successive accelerator, suggesting that the foil on the cylinder was not sufficiently uniform. For actual operation, we might require a feedback system to compensate for the fluctuation in the foil thickness.

Next, we started developing gas strippers. A gas stripper is free from lifetime-related problems, although it has a lower equilibrium charge state than a carbon foil because of the absence of the density effect. We did not have data on the equilibrium charge state in $\mathrm{N}_{2}$ gas, and no empirical formulas are available for its precise prediction. Therefore, we measured it at $11 \mathrm{MeV} /$ nucleon using a gas target system with a differential pumping system, which was formerly used for nuclear physics experiments [20]. The measured equilibrium charge state in $\mathrm{N}_{2}$ gas was $56+$, which is far below that in a carbon foil $(71+)$, suggesting that the gas stripper cannot be used for uranium beam acceleration because the minimum acceptable charge state for the fRC is $69+$.

However, the advantage of a gas stripper in being free from lifetime-related problems motivated us to research a way to achieve a higher charge state even in gases. The first solution is to increase the stripping energy because the equilibrium charge state generally increases as a function of the projectile energy. We measured equilibrium charge states at 14 and $15 \mathrm{MeV} /$ nucleon using the $\mathrm{N}_{2}$ gas stripper described above and found them to be $61+$ and $62+$, respectively [21]. An extrapolation of the results suggests that the stripping energy should be increased to $20 \mathrm{MeV} /$ nucleon in order to obtain an equilibrium charge state higher than $69+$, which is the lowest acceptable charge state for the fRC; however, the present stripping energy is $11 \mathrm{MeV} /$ nucleon. To realize the stripping at $20 \mathrm{MeV} /$ nucleon in our acceleration scheme, we need an additional accelerator of approximately $61 \mathrm{MV}$ before the stripper and a decelerator of approximately $31 \mathrm{MV}$ behind the stripper. Such extensive remodeling will cost more than U.S. $\$ 10$ million. The second solution is to change the stripping material to a low- $\mathrm{Z}\left(\mathrm{He}\right.$ or $\left.\mathrm{H}_{2}\right)$ gas, as will be discussed in the following sections.

\section{EQUILIBRIUM CHARGE STATE IN LOW-Z GAS}

Equilibrium charge state is determined by competition between the e-loss and e-capture processes of ions. The capture cross sections depend strongly on the ion velocity $V_{p}$ compared with that of the target electrons. The e-capture phenomenon is particularly highly suppressed because of poor kinematical matching when the ion 
TABLE II. Atomic collision systems for which enhancement of equilibrium charge is observed in a low- $Z$ target region. The parameter of $V_{p} / V_{1 s}$ is the relative projectile velocity with respect to K-shell electrons. The lower part of the table lists the reactions for which equilibrium charge-state measurements were carried out by our group in this study and in our previous studies $[20,21]$.

\begin{tabular}{lccc}
\hline \hline Reaction & $\begin{array}{c}\text { Energy } \\
(\mathrm{MeV} / \text { nucleon })\end{array}$ & $V_{p} / V_{1 s}$ & Reference \\
\hline $\mathrm{Ar}+\mathrm{H}_{2}$ & 1.25 & 7.1 & {$[22]$} \\
$\mathrm{U}+\mathrm{He}$ & 22 & 14.9 & {$[23]$} \\
$\mathrm{U}+\mathrm{N}_{2}$ & 56 & 6.8 & {$[24]$} \\
\hline $\mathrm{U}+\mathrm{He}$ & 11 & 10.5 & \\
$\mathrm{U}+\mathrm{He}$ & 14 & 11.9 & \\
$\mathrm{U}+\mathrm{He}$ & 15 & 12.3 & \\
$\mathrm{U}+\mathrm{N}_{2}$ & 11 & 3.0 & {$[20]$} \\
$\mathrm{U}+\mathrm{N}_{2}$ & 14 & 3.4 & {$[21]$} \\
$\mathrm{U}+\mathrm{N}_{2}$ & 15 & 3.5 & {$[21]$} \\
\hline \hline
\end{tabular}

velocity significantly exceeds the velocity of $1 s$ electrons, $V_{1 s}$, which are the fastest target electrons. Such suppression of e-capture is expected in cases of low- $Z$ targets or high ion velocity, because $V_{1 s}$ is approximately expressed by $Z / 137$, resulting in a higher equilibrium charge state. In fact, certain experimental data on the equilibrium charge state or effective charge at energies of $1-100 \mathrm{MeV} /$ nucleon in low- $Z$ strippers show a substantial increase in the equilibrium charge state [22-24]. Table II summarizes the reaction conditions under which charge enhancement of the equilibrium charge state in a low- $Z$ stripper is observed, along with the parameters of $V_{p} / V_{1 s}$ (the relative projectile velocities with respect to K-shell electrons) from [22-24]. This table also lists the parameters for the reactions for which the cross-section measurements were performed in He to obtain equilibrium charge states. These parameter values show that charge enhancement can be expected in our target reaction [U + He (gas) at $11 \mathrm{MeV} /$ nucleon]. The table further lists the parameters for reactions for which equilibrium charge states were measured in $\mathrm{N}_{2}$ gas; lower charge states than those in carbon foils are obtained in our previous study owing to the absence of the density effect [20,21].

Figure 3 shows e-loss and e-capture cross sections $\left(\sigma_{\mathrm{BEM}}^{L}\right.$ and $\left.\sigma_{\mathrm{SCH}}^{C}\right)$, calculated using the binary encounter model [25] and Schlachter's formula [26], as a function of the charge state for gases of $\mathrm{H}_{2}, \mathrm{He}$, and $\mathrm{N}_{2}$. The lines of these cross sections intersect at the equilibrium charge state. They clearly show higher equilibrium charge states in low- $Z$ gases than in $\mathrm{N}_{2}$ gas. There are no data on the equilibrium charge state of uranium ion in low- $Z$ gases in this energy region, mainly because of the difficulty in accumulating low- $Z$ gas without a window. For example, our gas stripper system, described in Sec. II, can accumulate only $0.015 \mathrm{mg} / \mathrm{cm}^{2}$ of $\mathrm{He}$ gas, which is not sufficient

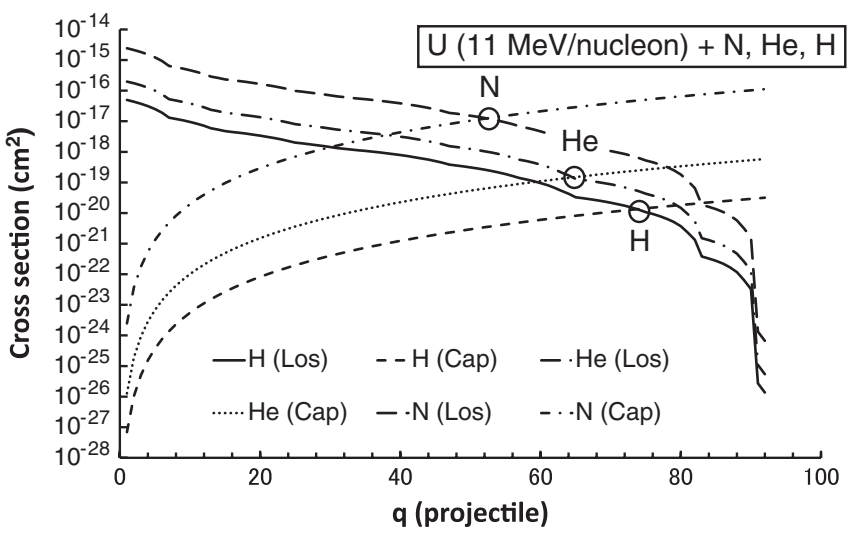

FIG. 3. Simple estimation of cross sections of e-loss and e-capture $\left(\sigma_{\mathrm{BEM}}^{L}\right.$ and $\left.\sigma_{\mathrm{SCH}}^{C}\right)$ for uranium beam in the gases of $\mathrm{N}_{2}$, He, and $\mathrm{H}_{2}$, calculated as a function of the charge state by using the binary encounter model [25] and Schlachter's formula [26]. The circles indicate the intersections of the two lines.

for uranium ions to attain equilibrium at $11 \mathrm{MeV} /$ nucleon; however, it can accumulate $1.3 \mathrm{mg} / \mathrm{cm}^{2}$ of $\mathrm{N}_{2}$ gas. Hence, we measured the cross sections of e-loss and e-capture as a function of the charge state of uranium ions in order to extract the equilibrium charge from their intersections.

The experiment was conducted at the RIBF using the RILAC and RRC. A schematic of the experimental setup is shown in Fig. 4. Beams of $11-\mathrm{MeV} /$ nucleon ${ }^{238} \mathrm{U}^{35+}$,

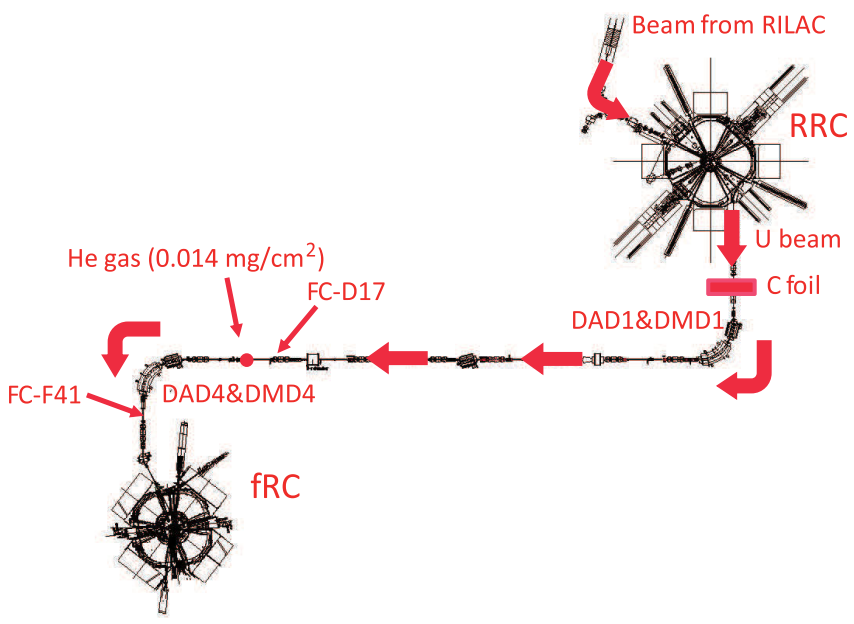

FIG. 4. Schematic of experimental setup for the measurements of cross sections of e-loss and e-capture for uranium beams at 11 , 14, and $15 \mathrm{MeV} /$ nucleon in RIBF beam lines between RRC and fRC. Uranium beams emerging from RRC passed through a carbon foil located in front of a pair of bending magnets (DAD1\&DMD1), which were used to select the individual projectile charge state. After emerging from the He gas cell, the beams passed through the second bending magnet (DAD4\&DMD4) into the Faraday cup at point F41 (FC-F41), which measured the intensity of the beam current of the charge state for e-loss, e-capture, no reaction, and so on. The Faraday cup at D17 (FC-D17) measured the current of the incoming ion with the aim of eliminating its fluctuation. 


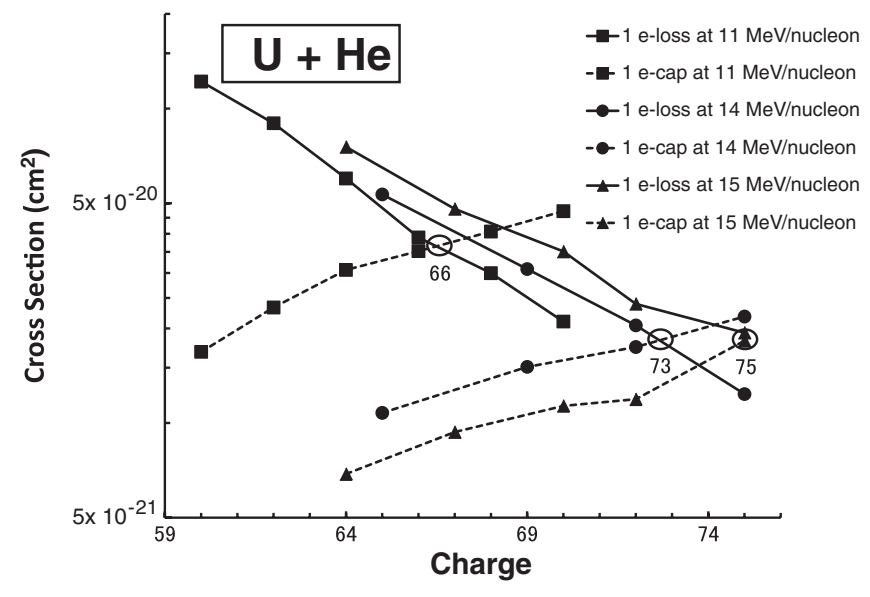

FIG. 5. Measured cross sections of e-loss and e-capture as a function of charge state of uranium ions at 11, 14, and $15 \mathrm{MeV} /$ nucleon in $\mathrm{He}$ gas. The cross sections were extracted assuming the thickness of the gas cell to be $13.27 \mu \mathrm{g} / \mathrm{cm}^{2}$. The intersections between the two lines for the cross sections of eloss and e-capture are indicated by the circles with the corresponding numbers of ion charge below.

$14-\mathrm{MeV} /$ nucleon ${ }^{238} \mathrm{U}^{41+}$, and $15-\mathrm{MeV} /$ nucleon ${ }^{238} \mathrm{U}^{41+}$ were extracted from the RRC. The incoming ions passed through a carbon foil located in front of a pair of bending magnets (DAD1\&DMD1); the carbon foil was used to select the individual projectile charge state $Q_{i}$. The thickness of the carbon foil was optimized so as to obtain the maximum intensity of the charge state. Each beam was directed through a windowless, differentially pumped He gas cell. After emerging from the gas cell, the beams passed through another pair of bending magnets (DAD4\&DMD4) into a Faraday cup at point F41 (FC-F41). The FC-F41 measured the intensity of the beam current of the charge state for e-loss $\left(Q_{i}+1\right)$, e-capture $\left(Q_{i}-1\right)$, no reaction $\left(Q_{i}\right)$, and so on. The pressure of the target He gas was monitored using a Baratron pressure transducer, and the gas flow was regulated by means of an automated control valve and flow controller. Further details of the experimental setup are given in Ref. [20]. The cross section of e-loss $\left(\sigma^{L}\right)$ and e-capture $\left(\sigma^{C}\right)$ was obtained using the following equations:

$$
\begin{gathered}
\sigma^{L}=-\frac{1}{n t}\left[\log \left(1-\frac{I_{F 41}\left(Q_{i}+1\right) /\left(Q_{i}+1\right) / I_{\mathrm{D} 17}}{\sum I\left(Q_{m}\right) / Q_{m} / I_{\mathrm{D} 17}}\right)\right]=\frac{R^{L}}{n t} \\
\sigma^{C}=-\frac{1}{n t}\left[\log \left(1-\frac{I_{F 41}\left(Q_{i}-1\right) /\left(Q_{i}-1\right) / I_{\mathrm{D} 17}}{\sum I\left(Q_{m}\right) / Q_{m} / I_{\mathrm{D} 17}}\right)\right]=\frac{R^{C}}{n t},
\end{gathered}
$$

where $n$ is the molecular number of He gas per unit weight; $t$, the gas thickness; $I_{F 41}(Q)$, the beam intensity of ion charge $Q$ at F41; and $I_{\mathrm{D} 17}$, the beam intensity of ion charge $Q_{i}$ at D17 upstream of the gas cell. $R^{L}$ and $R^{C}$ are reaction rates for e-loss and e-capture, respectively. The intensity at F41 was normalized by $I_{\mathrm{D} 17}$ to eliminate the fluctuation in the intensity of the beam from the RRC. During the measurements of the cross sections, the cell pressure was maintained at $0.56 \mathrm{kPa}$. At this pressure, the thickness of the gas stripper was measured to be $13.27 \pm 1.81 \mu \mathrm{g} / \mathrm{cm}^{2}$, using $\alpha$ rays from ${ }^{241} \mathrm{Am}$.

Figure 5 and Table III show the measured cross sections and the reaction rates as a function of the charge number of the uranium ions, respectively, at 11,14 , and $15 \mathrm{MeV} /$ nucleon. We assume that errors of $R^{L}$ and $R^{C}$ mainly originate from the reading errors for beam currents in a picoammeter and from the contribution of double

TABLE III. Experimental data on the cross sections of e-loss $\left(\sigma^{L}\right)$ and e-capture $\left(\sigma^{C}\right)$ and the reaction rates of e-loss $\left(R^{L}\right)$ and

\begin{tabular}{|c|c|c|c|c|c|}
\hline $\begin{array}{l}\text { Energy } \\
(\mathrm{MeV} / \text { nucleon})\end{array}$ & Charge & $R^{L}$ & $\begin{array}{c}\sigma^{L} \\
\left(\mathrm{~cm}^{2}\right) \\
\end{array}$ & $R^{C}$ & $\begin{array}{c}\sigma^{C} \\
\left(\mathrm{~cm}^{2}\right) \\
\end{array}$ \\
\hline 11 & $\begin{array}{l}60+ \\
62+ \\
64+ \\
66+ \\
68+ \\
70+\end{array}$ & $\begin{array}{l}0.216 \pm 0.012 \\
0.164 \pm 0.006 \\
0.113 \pm 0.003 \\
0.075 \pm 0.001 \\
0.058 \pm 0.001 \\
0.041 \pm 0.000\end{array}$ & $\begin{array}{l}1.219 \times 10^{-19} \\
8.980 \times 10^{-20} \\
5.996 \times 10^{-20} \\
3.894 \times 10^{-20} \\
2.998 \times 10^{-20} \\
2.103 \times 10^{-20} \\
\end{array}$ & $\begin{array}{l}0.033 \pm 0.000 \\
0.045 \pm 0.001 \\
0.059 \pm 0.001 \\
0.068 \pm 0.001 \\
0.078 \pm 0.001 \\
0.090 \pm 0.001\end{array}$ & $\begin{array}{l}.684 \times 10^{-20} \\
2.331 \times 10^{-20} \\
3.068 \times 10^{-20} \\
3.522 \times 10^{-20} \\
4.064 \times 10^{-20} \\
4.719 \times 10^{-20}\end{array}$ \\
\hline 14 & $\begin{array}{l}65+ \\
69+ \\
72+ \\
75+ \\
\end{array}$ & $\begin{array}{l}0.101 \pm 0.002 \\
0.060 \pm 0.002 \\
0.040 \pm 0.001 \\
0.024 \pm 0.000\end{array}$ & $\begin{array}{l}5.333 \times 10^{-20} \\
3.088 \times 10^{-20} \\
2.045 \times 10^{-20} \\
1.236 \times 10^{-20} \\
\end{array}$ & $\begin{array}{l}0.021 \pm 0.000 \\
0.030 \pm 0.000 \\
0.034 \pm 0.000 \\
0.043 \pm 0.000\end{array}$ & $\begin{array}{l}1.077 \times 10^{-20} \\
1.509 \times 10^{-20} \\
1.745 \times 10^{-20} \\
2.181 \times 10^{-20} \\
\end{array}$ \\
\hline 15 & $\begin{array}{l}64+ \\
67+ \\
70+ \\
72+ \\
75+\end{array}$ & $\begin{array}{l}0.140 \pm 0.003 \\
0.091 \pm 0.001 \\
0.068 \pm 0.001 \\
0.047 \pm 0.001 \\
0.038 \pm 0.001\end{array}$ & $\begin{array}{l}7.554 \times 10^{-20} \\
4.798 \times 10^{-20} \\
3.512 \times 10^{-20} \\
2.393 \times 10^{-20} \\
1.937 \times 10^{-20}\end{array}$ & $\begin{array}{l}0.014 \pm 0.000 \\
0.019 \pm 0.000 \\
0.033 \pm 0.000 \\
0.024 \pm 0.000 \\
0.036 \pm 0.000\end{array}$ & $\begin{array}{l}6.887 \times 10^{-21} \\
9.365 \times 10^{-21} \\
1.134 \times 10^{-20} \\
1.191 \times 10^{-20} \\
1.826 \times 10^{-20}\end{array}$ \\
\hline
\end{tabular}
e-capture $\left(R^{C}\right)$ for each charge of uranium beam in the energies of 11,14 , and $15 \mathrm{MeV} /$ nucleon. 
TABLE IV. Equilibrium charge state of uranium ion $Q_{e}$ in the gases of $\mathrm{He}, \mathrm{N}_{2}$, and $\mathrm{C}$ at 11,14 , and $15 \mathrm{MeV} /$ nucleon. The data for He gas were extracted from the intersections of the lines for the measured cross sections of e-loss and e-capture. The data for $\mathrm{N}_{2}$ gas and a carbon foil were obtained from Refs. [20,21].

\begin{tabular}{lccc}
\hline \hline Material & $\begin{array}{c}Q_{e} \text { at } 11 \\
(\mathrm{MeV} / \text { nucleon })\end{array}$ & $\begin{array}{c}Q_{e} \text { at } 14 \\
(\mathrm{MeV} / \text { nucleon })\end{array}$ & $\begin{array}{c}Q_{e} \text { at } 15 \\
(\mathrm{MeV} / \text { nucleon })\end{array}$ \\
\hline $\mathrm{He}$ & $66+$ & $73+$ & $75+$ \\
$\mathrm{N}_{2}$ & $56+$ & $61+$ & $62+$ \\
$\mathrm{C}$ & $72+$ & $76+$ & $77+$ \\
\hline \hline
\end{tabular}

scatterings, which can be expressed by $R_{Q}^{L}\left(R_{Q+1}^{L}+R_{Q+1}^{C}\right)$ or $R_{Q}^{C}\left(R_{Q-1}^{C}+R_{Q-1}^{L}\right)$. The absolute values of the cross section given in Table III have a deviation of $13.6 \%$ owing to the error of the target thickness; however, the relations between all the values for the cross sections have an accuracy similar to that of $R^{L}$ and $R^{C}$ because these values were extracted through scaling of $R^{L}$ and $R^{C}$ by constant $1 / n t$, assuming the thickness $t$ to be the mean of the measurement values $\left(13.27 \mu \mathrm{g} / \mathrm{cm}^{2}\right)$ described above. The data show that the cross section of the e-capture largely depends on the energy while that of e-loss does not depend much on the energy. Because the contribution of multiple electron transfer to the entire ionization process in He gas is very small [27], the intersection of the two lines for e-loss and e-capture provides a good approximation of the equilibrium charge state. The intersections are extracted to be $66+$, $73+$, and $75+$ at 11,14 , and $15 \mathrm{MeV} /$ nucleon, respectively. Table IV lists the equilibrium charge states in He gas, $\mathrm{N}_{2}$ gas, and a carbon foil. The equilibrium charge state in $\mathrm{He}$ gas is obviously larger than that in $\mathrm{N}_{2}$ gas by more than 10 and is close to that in a carbon foil.

\section{LOW-Z GAS STRIPPER}

The measurement results described in the previous section illustrate that a low- $Z$ gas stripper can be utilized to obtain the higher charge state of uranium. However, the difficulty in the accumulation of low- $Z$ gas due to its rapid dispersion still remains. In this section, we first present results of a simulation for attaining the necessary thickness of He gas to obtain a higher charge state. Next, we discuss two solutions for accumulating He gas up to the required thickness. The first solution involves the use of a standard differential pumping system, and the second involves the use of PWs (plasma windows) invented by Hershcovitch [28].

A simulation program was developed to demonstrate how $\mathrm{He}$ gas should be accumulated up to the required thickness to achieve the desired charge state. The rate equation in (3) was integrated over the thickness of $\mathrm{He}$ gas for the initial charge state of $35+$ :

$$
\frac{d P_{Q}(E)}{d t}=n\left[P_{Q-1} \sigma_{Q-1}^{L}-P_{Q}\left(\sigma_{Q}^{L}+\sigma_{Q}^{C}\right)+P_{Q+1} \sigma_{Q+1}^{C}\right],
$$

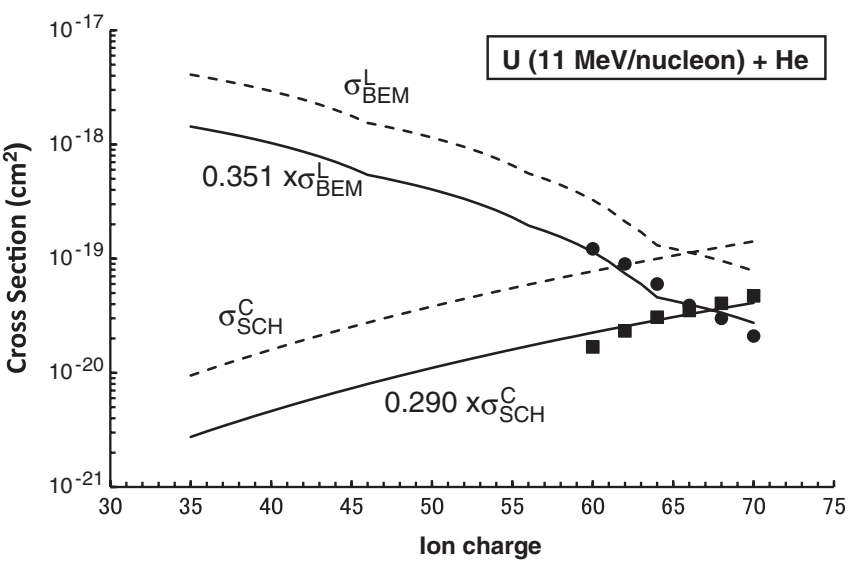

FIG. 6. Cross sections used for simulation of charge evolution of uranium ions in $\mathrm{He}$ gas at $11 \mathrm{MeV} /$ nucleon along with thickness. The dotted lines show the calculated cross sections of e-loss and e-capture $\left(\sigma_{\mathrm{BEM}}^{L}\right.$ and $\left.\sigma_{\mathrm{SCH}}^{C}\right)$ using the binary encounter model and Schlachter's formula. The solid lines show the scaled cross sections by 0.351 and 0.290 for e-loss and e-capture, respectively, in order to fit to the measured cross sections. The circles and squares denote the measured cross sections of e-loss and e-capture, respectively.

where $E$ is the projectile energy and $P_{Q}$ is the fraction of a charge state of $Q$. Figure 6 shows the cross sections for stripping and capture used in the simulation, defined as follows:

$$
\begin{aligned}
& \sigma_{Q}^{L}(E)=\alpha \sigma_{\mathrm{BEM}}^{L} \\
& \sigma_{Q}^{C}(E)=\beta \sigma_{\mathrm{SCH}}^{C} .
\end{aligned}
$$

The $\alpha$ in (4) and $\beta$ in (5) were determined to be 0.351 and 0.290 , respectively, through the fitting of the cross sections used in the prediction of the equilibrium charge states to the measured cross sections. The simulation includes just a single electron process, which provides good approximation in a low- $Z$ target as described in [27]. The program can include the energy loss of the ion through $\mathrm{He}$ gas, which is estimated using ATIMA [29]. Figure 7 shows the evolution of the mean charges as a function of gas thickness with and without energy loss of the projectile, obtained from the simulation. The mean charge without the energy loss monotonically goes up to the equilibrium charge state. The graph indicates that charge does not attain equilibrium even at $3 \mathrm{mg} / \mathrm{cm}^{2}$. However, the mean charge with energy loss starts falling around $1.75 \mathrm{mg} / \mathrm{cm}^{2}$, owing to the decrease in the equilibrium charge state as a function of the ion energy. This phenomenon indicates a slow ionization process in $\mathrm{He}$ gas wherein one electron dominates the process; however, in carbon foils, effective ionization occurs by virtue of the density effect. Though the mean charge reaches a maximum of approximately $65+$ at around $1.75 \mathrm{mg} / \mathrm{cm}^{2}$, practically, a thickness of less than $1 \mathrm{mg} / \mathrm{cm}^{2}$ is preferable because a thin stripper will induce less emittance growth due to multiple scattering and energy 


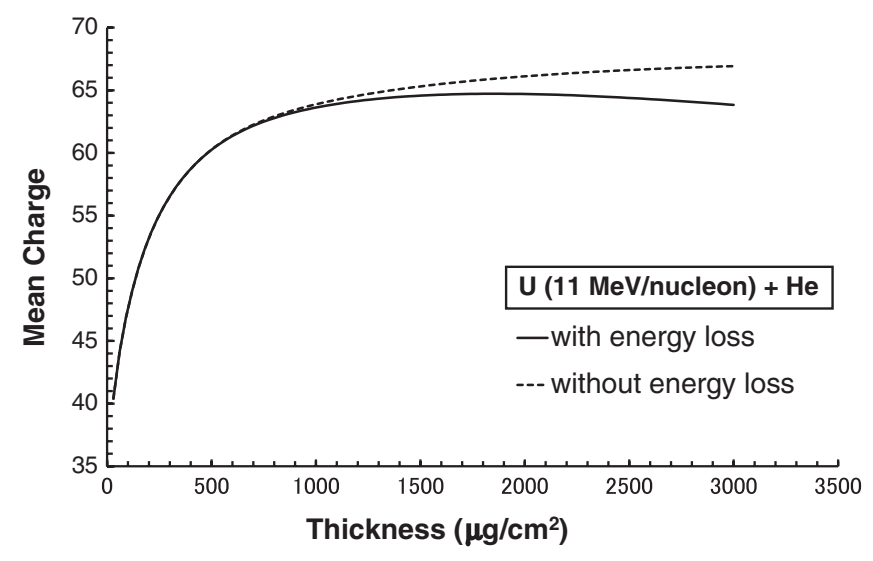

FIG. 7. Mean charge evolution of uranium ions at $11 \mathrm{MeV} /$ nucleon with and without energy loss of the projectile as a function of thickness of He gas.

straggling due to charge-state fluctuation [30,31]. The exact charge distribution will be measured in order to estimate the extent of remodeling of the fRC required [32].

Finally, we discuss how to accumulate about $1 \mathrm{mg} / \mathrm{cm}^{2}$ of He gas despite its rapid dispersion. Table V summarizes the specifications of the two solutions for the accumulation. The first solution is based on a three-stage standard differential pumping system with flow restrictors employed between the stages. The sophisticated design of this system [32] shows that a pressure of 0.2 atm is attainable in the gas cell, accumulating $1 \mathrm{mg} / \mathrm{cm}^{2}$ of He gas with a cell length of $40 \mathrm{~cm}$. The pump speeds of the mechanical booster pump (MBP) and the two turbo molecular pumps (TMPs) are $4200 \mathrm{~m}^{3} / \mathrm{h}, 2000 \mathrm{l} / \mathrm{s}$, and $1500 \mathrm{l} / \mathrm{s}$, respectively. The system requires an He gas flow of $10 \mathrm{Nm}^{3} / \mathrm{h}$ to maintain the pressure at $0.2 \mathrm{~atm}$. We require a system for recirculating the $\mathrm{He}$ gas flow because disposal of the $\mathrm{He}$ gas after its use in the gas cell would incur a daily cost of

TABLE V. Comparison between the gas stripper systems based on the plasma window (PW) and the big mechanical booster pump (MBP). $P, L$, and $A$ denote pressure, length, and aperture of the gas cell, respectively. The listed flow rate is that required to maintain the pressure in the gas cell. Components for connecting with the beam vacuum in differential pumping are also listed. Their pump speeds are listed in brackets, in $\mathrm{m}^{3} / \mathrm{h}$ for MBP and in 1/s for turbo molecular pump (TMP). Cases 1 and 2 with PW correspond to pressures of 0.2 and $1 \mathrm{~atm}$, respectively.

\begin{tabular}{lccc}
\hline \hline Item & MBP & $\begin{array}{c}\text { PW } \\
\text { (Case 1) }\end{array}$ & $\begin{array}{c}\text { PW } \\
\text { (Case 2) }\end{array}$ \\
\hline$P(\mathrm{~atm})$ & 0.2 & 0.2 & 1.0 \\
$L(\mathrm{~cm})$ & 40 & 40 & 8 \\
$A(\mathrm{~mm})$ & 6 & 6 & 6 \\
Flow rate $\left(\mathrm{Nm}^{3} / \mathrm{h}\right)$ & 10 & 0.0432 & 0.216 \\
Component for & MBP (4200) & MBP (400) & MBP (684) \\
differential pumping & TMP (2000) & TMP (190) & TMP (326) \\
& TMP (1500) & TMP (143) & TMP (244) \\
\hline \hline
\end{tabular}

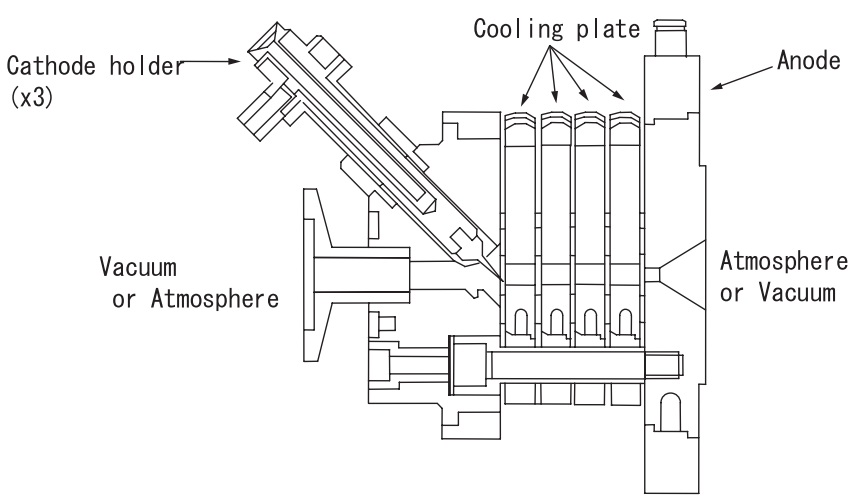

FIG. 8. Schematic of plasma window for keeping the vacuum separated from atmosphere. It consists of three cathode holders, cooling plates, and an anode plate to fill the aperture with arc plasma.

U.S. \$4000. Each component of the system is rather large but commercially available. The second solution is based on the PW, which is a wall-stabilized plasma arc used as an interface between the accelerator vacuum and pressurized targets. No solid material is introduced into the beam, and therefore, the PW can transmit a charged particle beam with low energy loss. It mainly consists of three cathodes, an anode, and some cooling plates to cool the plasma arc, as shown in Fig. 8. The arc in the PW can generate a pressure difference between its ends. Hence, it can maintain the pressure inside the gas cell while maintaining vacuum outside. Figure 9 shows a schematic of the low- $Z$ gas stripper containing two PWs, as suggested by Thieberger of BNL at the workshop on the charge stripper for FRIB in 2009 [33]. The low- $Z$ gas is accumulated in the cell, which is sandwiched between the two PWs. Table V lists the specifications of the system with the PWs, which achieves the same pressure as that achieved by the large differential pumping system. In this system, the flow rate required to maintain the pressure in the gas cell is as small as $0.0432 \mathrm{Nm}^{3} / \mathrm{h}$, determined on the basis of the operational experiences of Hershcovitch; this rate is about $1 / 1000$ of that required in the large differential pumping

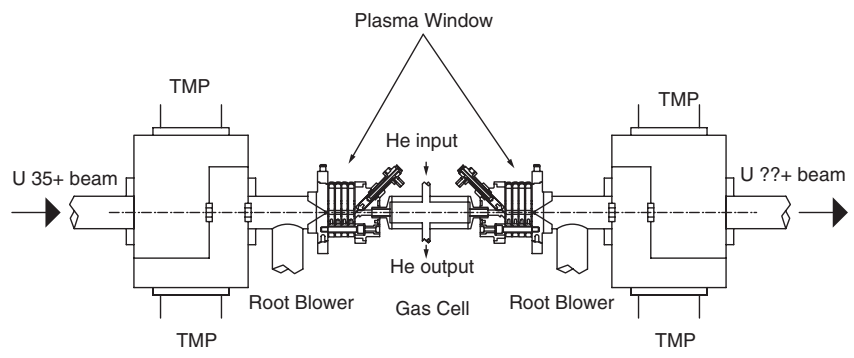

FIG. 9. Conceptual illustration of low- $Z$ gas stripper for $\mathrm{U}^{35+}$ beam with a pair of plasma windows (PWs). A gas cell with $\mathrm{He}$ input/output is located between the PWs. A pair of a root blower and two TMPs are located to connect the PWs with background vacuum for uranium beam. 
system mentioned above. The differential pumping system after the PW requires considerably smaller vacuum pumps than those in standard differential pumping systems. The experimental data demonstrate that the PW can accumulate more than $1 \mathrm{~atm}$ of $\mathrm{Ar}$ or air [34,35]. Table $\mathrm{V}$ also describes the system exploiting the merits of the PW, where the pressure in the $\mathrm{He}$ gas cell is 1 atm with the length of $8 \mathrm{~cm}$. It is easier to transport the beams passing through such a short gas cell sandwiched by a pair of flow restrictors with a small aperture.

\section{SUMMARY}

The operation of the RIBF from 2007 to 2010 was highly successful after the first beam was extracted. The new $28-\mathrm{GHz}$ superconducting ECR ion source and the new injector are ready for operation to increase the intensity of uranium beams. Recently, we found that a low- $Z$ gas stripper is a promising candidate for solving the as-yet unresolved stripper problem for high-power uranium beams. Measurement results showed that the equilibrium charge state in $\mathrm{He}$ gas is higher than that in $\mathrm{N}_{2}$ gas by more than 10 .

\section{ACKNOWLEDGMENTS}

The authors would like to acknowledge the cyclotron crews for providing high-quality beams and for their help during preparation and measurements. The authors express gratitude to Dr. T. Azuma for the useful discussions regarding the phenomena of ionization and electron capture in low- $Z$ gases. The authors express their gratitude to Dr. F. Marti for the useful discussions during the experiment.

[1] Y. Yano, Nucl. Instrum. Methods Phys. Res., Sect. B 261, 1009 (2007).

[2] M. Kase et al., in Proceedings of the 17th International Conference on Cyclotrons and their Applications, Yoyogi, Tokyo, 2004, p. 160.

[3] M. Odera et al., Rev. Sci. Nucl. Instrum. Methods A 227, 4523 (1999).

[4] A. Goto et al., Proceedings of the 12th International Conference on Cyclotrons and their Applications, Berlin (1989), p. 51.

[5] N. Inabe et al., in Proceedings of the 17th International Conference on Cyclotrons and their Applications, Yoyogi, Tokyo, 2004, p. 200.
[6] J. Ohnishi et al., in Proceedings of the 17th International Conference on Cyclotrons and their Applications, Yoyogi, Tokyo, 2004, p. 197.

[7] H. Okuno et al., IEEE Trans. Appl. Supercond. 18, 226 (2008).

[8] T. Kubo, Nucl. Instrum. Methods Phys. Res., Sect. B 204, 97 (2003).

[9] N. Fukunishi and J. Particle, J. Part. Accel. Soc. Jpn. 4, 112 (2007).

[10] A. Goto et al., in Proceedings of the 18th International Conference on Cyclotrons and their Applications, Giardini Naxos, Italy, 2007, p. 3.

[11] T. Ohnishi et al., J. Phys. Soc. Jpn. 79, 073201 (2010).

[12] T. Nakamura et al., Phys. Rev. Lett. 103, 262501 (2009).

[13] P. Doornenbal et al., Phys. Rev. Lett. 103, 032501 (2009).

[14] T. Nakagawa et al., High Energy Phys. Nucl. Phys. 31, 133 (2007).

[15] J. Ohnishi et al., High Energy Phys. Nucl. Phys. 31, 37 (2007).

[16] O. Kamigaito et al., in the 3rd Annual Meeting of PASJ and 31st Linac Meeting in Japan, Sendai, 2006, p. 502.

[17] ACF-Metals Arizona Carbon Foil Co, Inc., http://www.techexpo.com/firms/acf-metal.html.

[18] H. Hasebe et al., Nucl. Instrum. Methods Phys. Res., Sect. A 613, 453 (2010).

[19] H. Ryuto et al., Nucl. Instrum. Methods Phys. Res., Sect. A 569, 697 (2006).

[20] H. Kuboki et al., Phys. Rev. ST Accel. Beams 13, 093501 (2010).

[21] H. Kuboki et al. (unpublished).

[22] T. Mizogawa et al., RIKEN Accel. Prog. Rep. 22, 62 (1988).

[23] R. Bimbot et al., Nucl. Instrum. Methods Phys. Res., Sect. B 107, 9 (1996).

[24] A. Fettouchi et al., Nucl. Instrum. Methods Phys. Res., Sect. B 245, 32 (2006).

[25] M. Gryzinski, Phys. Rev. 138, A305 (1965); 138, A322 (1965); 138, A336 (1965).

[26] A. S. Schlachter et al., Phys. Rev. A 27, 3372 (1983).

[27] H.-D. Betz, in Appl. At. Coll. Phys. 4, 1 (1983).

[28] A. Hershcovitch, J. Appl. Phys. 78, 5283 (1995).

[29] ATIMA, GSI, Germany, http://www-linux.gsi.de/weick/atima/.

[30] H. Weick et al., Phys. Rev. Lett. 85, 2725 (2000).

[31] H. Weick, A. H. Soerensen et al., Nucl. Instrum. Methods Phys. Res., Sect. B 193, 1 (2002).

[32] H. Imao et al. (unpublished).

[33] Facility for Rare Isotope Beams (FRIB), Stripper and Target Technology for High Power Heavy Ion Beams Workshop, East Lansing (2009).

[34] A. Hershcovitch, Phys. Plasmas 5, 2130 (1998).

[35] B. T. Pinkoski, A. Hershcovitch et al., Rev. Sci. Instrum., 721677 (2001). 